\title{
CARACTERIZAÇÃO FÍSICA E QUANTIDADE DE NUTRIENTES EM FRUTOS DE MARACUJÁ DOCE ${ }^{1}$
}

\author{
MARCO ANTONIO DA SILVA VASCONCELLOS ${ }^{2}$, EDSON TADASHI SAVAZAKI ${ }^{2}$, \\ HÉLIO GRASSI FILHO², RUBENS NEI BRIANÇON BUSQUET ${ }^{4}$, JOSÉ LUIZ MOSCA²
}

\begin{abstract}
RESUMO - O presente trabalho foi conduzido com o objetivo de avaliar algumas características físicas e a distribuição dos nutrientes nos frutos de maracujá doce (Passiflora alata Dryand.), segundo sua classificação. Os frutos classificados como tipo 8 foram maiores e mais pesados que os dos tipos 9 e 12, apresentando maior peso de casca e de polpa. Independentemente da classificação dos frutos, o N, P, B, Cu, Fe e Zn estão distribuídos em maior quantidade na polpa do que na casca dos frutos. O K, Ca, S e o Mn estão distribuídos em maior quantidade na casca do que na polpa dos frutos. O magnésio apresenta uma distribuição na casca levemente superior à da polpa. Na média dos três tipos de frutos, os valores obtidos por fruto (casca+polpa) foram: 578,6 mg de N; 81,9 mg de P; 740,6 mg de $\mathrm{K} ; 56,8 \mathrm{mg}$ de Ca; 64,6 mg de Mg; 98,3 mg de S; 452,3 mg de B; 302,5 mg de Cu; $1471 \mathrm{mg}$ de Fe; 167,5 mg de Mn, e 644,1 mg de Zn.
\end{abstract}

Termos de indexação: Passiflora alata, macronutrientes, micronutrientes, casca, polpa

\section{PHYSICAL CHARACTERIZATION AND QUANTITY OF NUTRIENTS IN SWEET PASSION FRUIT}

\begin{abstract}
The present work was conducted in Botucatu, state of São Paulo, in order to evaluate some physical characteristics and the nutrient distribution in sweet passion fruit (Passiflora alata Dryand.), by type. The fruit classified as type 8 were larger than those classified as type 9 and 12, have larger weight of peel and pulp. Independently of fruit classification, N, P, B, Cu, Fe, and Zn were found in larger quantities throughout the pulp than in the peel. $\mathrm{K}, \mathrm{Ca}, \mathrm{S}$, and $\mathrm{Mn}$ were present in greater quantity in the peel than in the pulp. $\mathrm{Mg}$ showed slightly larger presence in the peel. The fruits of sweet passion fruit presented, in average: $578.6 \mathrm{mg}$ of N; $81.9 \mathrm{mg}$ of $\mathrm{P}$; $740.6 \mathrm{mg}$ of K; $56.8 \mathrm{mg}$ of Ca; $64.6 \mathrm{mg}$ of Mg; $98.3 \mathrm{mg}$ of $\mathrm{S} ; 452.3 \mathrm{mg}$ of B; $302.5 \mathrm{mg}$ of Cu; $1471 \mathrm{mg}$ of Fe; $167.5 \mathrm{mg}$ of Mn; and $644.1 \mathrm{mg}$ of Zn.
\end{abstract}

Index terms: Passiflora alata, macronutrients, micronutrients, peel, pulp

\section{INTRODUÇÃO}

A expansão do cultivo do maracujazeiro doce não tem sido acompanhada pela geração tecnológica, fazendo com que, na maioria das vezes, o próprio produtor tenha que desenvolver técnicas para solucionar os problemas surgidos. Estas novas técnicas, geralmente, estão apoiadas nas informações disponíveis para o cultivo do maracujazeiro-amarelo (Passiflora edulis Sims f. flavicarpa Deg.).

De acordo com Vasconcellos \& Cerda (1994), os frutos do maracujazeiro doce, devido às características de sua polpa, são consumidos principalmente como fruta fresca, não sendo utilizados para elaboração de suco. A comercialização é feita em caixetas de papelão $(+/-3,0 \mathrm{Kg})$, que recebem a classificação por tipos, em função do número de frutos presentes no seu interior. Esse número varia comercialmente de 8 a 24. Quanto maior for a classificação por tipo, menor será então tamanho do fruto. Essa classificação garante ao produtor um preço diferenciado pelo produto vendido.

Por apresentar uma exploração econômica recente, observa-se no campo grandes variações nas características relacionadas à qualidade dos frutos, como formato, peso, cor da polpa, sabor e o amolecimento basal dentre outros. A fixação destas características é importante para viabilizar a identificação do consumidor com o produto e, conseqüentemente, o sucesso comercial de sua exploração.

Oliveira et al. (1982) relatam que os frutos do maracujazeiro doce apresentam as seguintes características: formato piriforme; peso de 80 a $300 \mathrm{~g} ; 200$ a 300 sementes/fruto; $62,10 \%$ de casca; $9,74 \%$ de suco; e $26,20 \%$ de polpa residual. Num estudo sobre o desenvolvimento de frutos do maracujá doce, Vasconcellos et al. (1993) relatam os seguintes valores: peso médio de 270g; comprimento médio de $9,91 \mathrm{~cm}$; largura média de $8,29 \mathrm{~cm} ; 290$ sementes/fruto, $24,22 \%$ de polpa e $75,78 \%$ de casca .

Comparando a qualidade dos frutos de dez procedências de maracujá doce, produzidas no Distrito Federal, Anselmo et al. (1998) observaram, dentre outras características: peso médio de fruto variando de 134 a $305 \mathrm{~g}$ e rendimento de suco variando de 19,6 a $24,0 \%$.

Com relação aos teores de nutrientes presentes nos frutos das diversas espécies do gênero Passiflora, a literatura é escassa, reportando-se principalmente ao maracujá-amarelo (Passiflora edulis Sims. f. flavicarpa Deg.) e maracujá-roxo (Passiflora edulis Sims). De acordo com Fernandes et al. (1977), o fruto do

1 (Trabalho 146/2000). Recebido: 17/07/2000. Aceito para publicação: 04/10/2001.

2 PósGraduando do curso de PG Horticultura da FCA/UNESP-Botucatu-SP (masv@laser.com.br) CX. Postal 237 DPV/Horticultura Botucatu - SP CEP 18603-970

3 Prof. Dr. FCA/UNESP - Botucatu - SP CX. Postal 237 Dep. Recursos Naturais Botucatu - SP CEP 18603-970

4 Prof. MSc. IA/DF, UFRRJ, Km 7 da BR465 Seropédica - RJ CEP 23851-970 (rbusquet@ufrrj.br); 
maracujazeiro-amarelo apresenta: $350 \mathrm{mg}$ de $\mathrm{N} ; 27 \mathrm{mg}$ de $\mathrm{P} ; 350$ mg de K; 19 mg de Ca; 19 mg de Mg; 30 mg de S; 194 mg de B; 72 $\mathrm{mg}$ de $\mathrm{Cu} ; 718 \mathrm{mg}$ de Fe; $268 \mathrm{mg}$ de Mn e $1290 \mathrm{mg}$ de Zn.

Visando a um maior conhecimento sobre a quantidade de nutrientes presentes na composição das diferentes partes do fruto (casca e polpa (suco+sementes)), tendo em vista a importância das frutas como fonte de minerais na saúde humana, além da possibilidade de utilização de frutos que não apresentem padrão para comercialização e frutos atacados por pragas ou doenças para elaboração de ração animal ou de substratos, o presente trabalho foi conduzido com o objetivo de avaliar algumas características físicas do fruto do maracujá doce em função da sua classificação por tipo, bem como a distribuição dos nutrientes na casca e na polpa.

\section{MATERIAL E MÉTODOS}

No presente estudo, foram utilizados frutos de maracujá doce provenientes de um cultivo comercial localizado no município de Lins - SP. As plantas estavam no final do primeiro ano de produção, com uma produtividade de 15,7 t./ha $(31,5 \mathrm{~kg}$ de frutos por planta). $\mathrm{O}$ sistema de condução adotado foi o de espaldeira vertical, sendo o espaçamento de plantio de $4,0 \mathrm{~m} \mathrm{X}$ $5,0 \mathrm{~m}$ e a adubação e correção de solo realizadas segundo as recomendações de Raij et al. (1996).

Para a análise das características físicas e da distribuição dos nutrientes, os frutos foram classificados por tipos ( $8 ; 9$ e 12), de acordo com seu tamanho, sendo retirados 10 frutos ao acaso para avaliação de: comprimento e largura; peso do fruto; peso da matéria fresca e seca da casca e da polpa (semente+suco); e porcentagem de água na casca e na polpa.

A análise das concentrações dos nutrientes foi realizada segundo a metodologia descrita por Malavolta et al. (1989), utilizando 10 frutos de cada tipo, selecionados ao acaso, divididos em casca e polpa, colocados para secar em estufa por 72 horas . No caso da polpa, o tempo de secagem foi de 5 dias para permitir posterior moagem do material.

Para cada nutriente, foi realizada a análise de variância dos dados e determinadas por comparação ortogonal as diferenças entre: Y1- quantidade dos nutrientes na polpa dos 3 tipos de frutos (Polpa 8 X Polpa 9 X Polpa 12); Y2- quantidade dos nutrientes na casca dos 3 tipos de frutos (Casca 8 X Casca 9 $\mathrm{X}$ Casca 12); Y3- quantidade média dos nutrientes nas polpas versus nas cascas (Polpas (8; 9 e 12) X Cascas (8; 9 e 12)); Y4quantidade dos nutrientes na polpa versus na casca do fruto tipo 8 (Polpa 8 X Casca 8); Y5- quantidade dos nutrientes na polpa versus na casca do fruto tipo 9 (Polpa 9 X Casca 9); Y6quantidade dos nutrientes na polpa versus na casca do fruto tipo 12 (Polpa 12 X Casca 12).

\section{RESULTADOS E DISCUSSÃO}

Os resultados das análises foliar e de solo do maracujazeiro doce obtidos no presente experimento são apresentados nas Tabelas 1 e 2. Estes valores, quando comparados com os valores relatados para o maracujazeiroamarelo por Baumgartner (1987), mostram que apenas o boro (com valores inferiores) e o cobre (com valores 6 vezes superiores) foram os nutrientes que apresentaram valores fora da faixa relatada por Baumgartner (1987).

Os dados apresentados na Tabela 3 mostram que os frutos do tipo 8 apresentaram comprimento e largura superiores aos frutos tipo $9 \mathrm{e} 12$. Como conseqüência desse maior crescimento, os frutos tipo 8 apresentaram peso médio superior ao dos frutos tipo 9 e 12 (18,23\% e $31,95 \%$, respectivamente), assim como maiores pesos de matéria fresca e seca de casca e polpa. Os frutos do tipo 8 apresentam maior porcentagem de peso de matéria fresca e menor de matéria seca da casca que os frutos tipo 9 e 12, mostrando a casca do fruto tipo 8 possuir maior porcentagem de água. De forma contrária, a \% de água na polpa dos frutos do tipo 8 foi menor que a da polpa dos frutos 9 e 12, reflexo do maior número de sementes formadas nos frutos do tipo 8 , levando a um maior peso de matéria fresca e seca de polpa, e não apenas de volume de suco formado. Os frutos, independentemente de sua classificação, apresentaram em média $90 \%$ de água na casca e $70 \%$ de água na polpa.

Os valores obtidos para os frutos tipo 8; 9 e 12 de: peso médio $(273,3 \mathrm{~g}, 223,4 \mathrm{~g}, 185,9 \mathrm{~g})$, peso da casca $(20,3 \mathrm{~g}, 17,5 \mathrm{~g}$ e $14,9 \mathrm{~g})$ e peso da polpa $(67,1 \mathrm{~g}, 60,2 \mathrm{~g}$ e $49,3 \mathrm{~g})$, estão dentro dos valores relatados por Oliveira et al. (1982), Vasconcellos et al. (1993) e por Anselmo et al. (1998).

Quanto ao comprimento e largura, os frutos do tipo 8 apresentaram valores médios de $11,60 \mathrm{~cm}$ e $8,03 \mathrm{~cm}$, respectivamente, que foram superiores aos observados por Vasconcellos et al. (1993), possivelmente devido ao formato dos frutos que, em sua maioria, apresentavam forma piriforme.

A análise de variância mostrou existir diferenças estatísticas para todos os macronutrientes, nos contrastes propostos.

Com relação à distribuição dos macronutrientes na casca e polpa dos frutos (Tabela 4), observa-se que, independentemente da classificação dos frutos, o nitrogênio estava em quantidades estatisticamente superiores na polpa em relação à casca, resultado este esperado por as sementes de maracujá possuírem cerca de $10-12 \%$ de proteína, além da sua importância na formação de aminoácidos, aminas e alcalóides. Por esse motivo, os frutos do tipo 8 diferem estatisticamente dos frutos 9 e 12 quanto à quantidade de nitrogênio na polpa por apresentarem maior número de sementes formadas. O mesmo ocorre com o fósforo, presente nas sementes na forma de fosfatos e ácidos nucléicos dentre outros, apresentando valores na polpa cerca de 2,5 vezes superiores aos da casca.

O potássio, cálcio, magnésio e enxofre estavam em concentrações significativamente superiores na casca, sendo que o cálcio, por estar presente na formação da parede celular e pectina, apresentou valores 3,0 a 3,5 vezes superiores na casca em relação à polpa. Em ambos os casos, essa relação diminui com a redução na qualidade do fruto. O potássio foi encontrado em quantidade estatisticamente superior na casca em relação à polpa, independentemente da classificação dos frutos. Contudo, a diferença entre a quantidade de potássio na casca e na polpa variou de $10-15$ a $30 \%$, sendo que os frutos tipo 12 apresentaram a maior diferença, reflexo do menor peso de polpa formada, confirmando a importância do potássio na qualidade dos frutos. $\mathrm{O}$ enxofre também esteve presente em quantidades estatisticamente superiores na casca em relação à polpa, 
TABELA 1 - Resultado da Análise foliar de plantas de maracujá doce cultivadas em Lins - SP, realizada pelo Laboratório de Nutrição Mineral de Plantas da FCA/UNESP - Botucatu.

\begin{tabular}{|c|c|c|c|c|c|c|c|c|c|c|}
\hline \multicolumn{5}{|c|}{$\mathrm{g} \cdot \mathrm{kg}-^{1}$} & \multicolumn{6}{|c|}{$\mathrm{mg} \cdot \mathrm{kg}^{-1}$} \\
\hline $\mathbf{N}$ & $\mathbf{P}$ & K & $\mathrm{Ca}$ & M g & $\mathbf{S}$ & B & $\mathrm{Cu}$ & $\mathrm{Fe}$ & M n & $Z_{n}$ \\
\hline 31 & 1,8 & 15 & 24 & 6,5 & 35 & 28 & 107 & 97 & 92 & 30 \\
\hline
\end{tabular}

TABELA 2 - Resultado da análise química do solo $(0$ - 20cm e 20 - 40cm) da área de cultivo com maracujá doce localizada em Lins - SP, realizada pelo Laboratório de Fertilidade do Solo da FCA/UNESP - Botucatu.

\begin{tabular}{|c|c|c|c|c|c|c|c|c|c|}
\hline Amostra & $\mathrm{pH} \mathrm{CaCl}{ }_{2}$ & $\begin{array}{l}\text { M. O. } \\
\text { g/dm }\end{array}$ & $\mathrm{Al}^{3+}$ & $\mathrm{K}$ & $\mathrm{Ca}$ & $\mathrm{Mg}$ & SB & CTC & $\mathrm{V} \%$ \\
\hline & & & ------- & --- & - & $\mathrm{mmol}_{\mathrm{c}} \mathrm{dm}^{3-1}$ & & -------- & ------ \\
\hline $0-20 \mathrm{~cm}$ & 5,7 & 10 & ------- & 2,5 & 38 & 13 & 53 & 66 & 80 \\
\hline $20-40 \mathrm{~cm}$ & 5,6 & 12 & -------- & 4,3 & 25 & 8 & 37 & 52 & 72 \\
\hline Amostra & B oro & \multicolumn{2}{|c|}{ Cobre } & \multicolumn{2}{|c|}{$\begin{array}{l}\text { Ferro } \\
\mathrm{mg} \cdot \mathrm{dm}^{3-1}\end{array}$} & \multicolumn{2}{|c|}{ Manganês } & \multicolumn{2}{|c|}{ Zinco } \\
\hline $0-20 \mathrm{~cm}$ & 0,04 & & 2,3 & \multicolumn{2}{|c|}{24} & \multicolumn{2}{|c|}{4,1} & \multicolumn{2}{|c|}{0,8} \\
\hline $20-40 \mathrm{~cm}$ & 0,05 & & 6,2 & \multicolumn{2}{|c|}{22} & \multicolumn{2}{|c|}{7,6} & \multicolumn{2}{|c|}{1,6} \\
\hline
\end{tabular}

TABELA 3 - Médias do comprimento, largura, peso total, peso e $\%$ de matéria fresca de casca e polpa, peso e $\%$ de matéria seca de casa e polpa, e \% de água na casca e na polpa de frutos de maracujá doce (Passiflora alata Dryand.), segundo sua classificação por tipo.

\begin{tabular}{|c|c|c|c|}
\hline$\underbrace{\text { Classifica çã }}_{\text {C aracterísticas }}$ & T ip o 8 & T ip o 9 & T ip o 12 \\
\hline Comprimento (cm) & 11,6 & 11,1 & 10,6 \\
\hline Largura (c m) & 8,0 & 7,5 & 7,0 \\
\hline Pesototal (g) & 273,3 & 223,5 & 185,9 \\
\hline $\begin{array}{r}\text { Peso }(g) \text { mat. } \\
\text { fresca da casca }\end{array}$ & 206,2 & 163,3 & 136,7 \\
\hline $\begin{array}{r}\text { Peso (g) mat. } \\
\text { fresca da polpa }\end{array}$ & 67,1 & 60,2 & 49,3 \\
\hline $\begin{array}{l}\text { Peso(g) mat. seca da } \\
\text { casca }\end{array}$ & 20,4 & 17,5 & 14,9 \\
\hline Peso $\underset{\text { d a polpa }}{(g) \text { mat. Seca }}$ & 20,1 & 17,3 & 14,2 \\
\hline$\%$ de águ a na casca & 90,1 & 89,3 & 89,0 \\
\hline$\%$ d e água na polpa & 70,0 & 71,3 & 71,1 \\
\hline
\end{tabular}


TABELA 4 - Médias da quantidade de macronutrientes (mg) distribuídos na casca e polpa (semente+suco) de frutos de maracujá doce (Passiflora alata Dryand.) em função de sua classificação por tipo.

\begin{tabular}{|c|c|c|c|c|}
\hline & & T ip o 8 & T ip o 9 & T ip o 12 \\
\hline \multirow{2}{*}{ Nitrogên io } & $\mathrm{C}$ a s c a & $290,6 \mathrm{bA}$ & 227,9 b B & 213,5 b B \\
\hline & P o lp a & 382,7 a $A$ & 332,8 a B & 288,2 a $C$ \\
\hline \multirow{2}{*}{ F ó s foro } & $\mathrm{C}$ a s c a & 28,5 b A & 28,0 b A & 24,0 b A \\
\hline & P o lp a & 65,5 a $A$ & 52,7 a B & 47,0 a B \\
\hline \multirow[b]{2}{*}{ Potás sio } & $\mathrm{C}$ a s c a & 504,7 a A & 374,5 a $\mathrm{B}$ & 328,5 a B \\
\hline & P o lp a & 438,3 b A & 337,4 b B & 238,4 b C \\
\hline \multirow[b]{2}{*}{ C á le io } & $\mathrm{C}$ a s c a & 40,9 a $B$ & 49,9 a $A$ & 40,8 a B \\
\hline & P o lp a & 15,1 b A & 12,5 b B & 11,1 b B \\
\hline \multirow[b]{2}{*}{ M a g nésio } & $\mathrm{C}$ a s c a & 39,8 a A & 33,8 a B & 28,9 a C \\
\hline & P o lp a & 35,8 b A & 29,8 b B & $25,6 \mathrm{~b} \mathrm{C}$ \\
\hline \multirow[b]{2}{*}{ Enxofre } & $\mathrm{C}$ a s c a & 72,9 a $\mathrm{A}$ & 55,2 a B & 50,9 a B \\
\hline & P o lp a & 47,3 b A & 36,3 b B & 32,4 b B \\
\hline
\end{tabular}

Para cada macronutriente:

*Letras minúsculas, iguais na coluna e maiúsculas iguais na linha, não diferem estatisticamente entre si, a 5\% de significância

*As médias nas colunas são referentes aos contrastes Y4, Y5 e Y6 e, nas linhas, aos contrastes Y1 e Y2

TABELA 5 - Médias da quantidade de micronutrientes $(\mu \mathrm{g})$ distribuídos na casca e polpa de frutos de maracujá doce (Passiflora alata Dryand.) em função de sua classificação por tipo.

\begin{tabular}{|c|c|c|c|c|}
\hline & & T ip o 8 & Tip o 9 & T ip o 12 \\
\hline \multirow{3}{*}{ B oro } & $\mathrm{C}$ a sc a & $229,4 \mathrm{bA}$ & 211,8 b B & $153,0 \mathrm{bC}$ \\
\hline & P o lp a & 297,1 a A & 241,0 a B & 224,7 a B \\
\hline & $\mathrm{C}$ a sc a & 152,9 bA & 144,6 b B & 119,8 a C \\
\hline \multirow{2}{*}{ Cobre } & P o lp a & 186,3 a A & 168,6 a B & 135,2 a C \\
\hline & $\mathrm{C}$ a sc a & $876,8 \mathrm{bA}$ & 644,2 bB & 558,0 a C \\
\hline \multirow{2}{*}{ Ferro } & P o lp a & 1022,1 a A & 739,2 aB & 572,8 a C \\
\hline & $\mathrm{C}$ a sca & 158,5 a A & 135,8 a B & 78,7 a C \\
\hline \multirow{2}{*}{ M anganês } & P o lp a & $55,4 \mathrm{bA}$ & 38,9 b B & $35,6 \mathrm{bB}$ \\
\hline & $\mathrm{C}$ a sc a & $137,6 \mathrm{bA}$ & 118,3 b B & $86,2 \mathrm{bC}$ \\
\hline Zinco & P o lp a & 639,4 a $A$ & 527,4 a B & 423,4 a C \\
\hline
\end{tabular}

Para cada macronutriente:

*Letras minúsculas, iguais na coluna e maiúsculas iguais na linha, não diferem estatisticamente entre si, a 5\% de significância

*As médias nas colunas são referentes aos contrastes Y4, Y5 e Y6 e, nas linhas, aos contrastes Y1 e Y2

independentemente da classificação do fruto (+/-35\%). Contudo, na comparação entre as quantidades presentes nas cascas dos três tipos de frutos, os frutos tipo 8 apresentaram quantidades estatisticamente superiores aos dos frutos tipo 9 e estes não diferiram dos frutos tipo 12, sendo o mesmo observado em relação à polpa. Do mesmo modo, o magnésio foi encontrado em maior quantidade na casca que na polpa dos frutos, independentemente de sua classificação, assim como na comparação entre casca e polpa por tipo de fruto, onde a diferença entre os valores da casca e polpa variou de 10 a $11,8 \%$.

De forma geral, os frutos do maracujazeiro doce, considerando a média dos três tipos de frutos classificados, apresentaram a seguinte quantidade de nutrientes (casca+polpa): 578,6 mg de $\mathrm{N} ; 81,9 \mathrm{mg}$ de P; 740,6 mg de K; 56,8 mg de Ca; 64,6 $\mathrm{mg}$ de $\mathrm{Mg}$; e 98,3 mg de S. Esses valores, para todos os macronutrientes, são diferentes dos obtidos por Fernandes et al. (1977) para o maracujazeiro-amarelo, com concentrações superiores, da ordem de $65,3 \% \mathrm{~N}, 300,0 \%$ P, 211,6 \% K, 298,9 $\% \mathrm{Ca}, 340,0 \% \mathrm{Mg}$ e $327,6 \% \mathrm{~S}$.

Para os micronutrientes avaliados, a análise de variância mostrou diferenças significativas nos contrastes propostos.

Os contraste Y1, Y2 e Y3 mostraram haver diferenças 
significativas para os micronutrientes boro, cobre, ferro, manganês e zinco, onde, tanto as polpas (contraste Y1) como as cascas (contraste Y2) dos frutos tipo 8, apresentaram valores significativamente superiores aos dos frutos 9 e 12 . A comparação entre as médias das polpas $\mathrm{X}$ as médias das cascas mostrou ocorrer diferenças significativas para o boro, cobre, ferro e o zinco, onde estes apresentaram maiores concentrações na polpa, enquanto o manganês foi encontrado em maiores concentrações na casca dos frutos. Nas comparações entre polpa X casca, para cada tipo de fruto, o cobre e o ferro não diferiram estatisticamente entre si nas quantidades presentes na polpa e casca dos frutos tipo 12.

O Quadro 5 apresenta os valores da distribuição dos micronutrientes na casca e polpa dos frutos, observando-se que o boro, cobre, ferro e zinco apresentaram maiores concentrações nas polpas dos frutos do que nas cascas para os tipos de frutos classificados. As maiores concentrações nas polpas e cascas foram observadas nos frutos tipo 8 , que diferiram estatisticamente dos frutos tipo 9 e estes dos frutos tipo 12, ressaltando que o cobre e o ferro não diferiram entre si para os frutos tipo 12. A comparação entre as quantidades de boro, cobre, ferro e zinco presentes na polpa dos três tipos de frutos mostrou que, quanto maior o fruto, maior a quantidade dos referidos micronutrientes. Deve ser ressaltado que a diferença nas quantidades de boro e zinco na polpa em relação à casca, entre os diferentes tipos de frutos classificados, é reflexo do maior peso de polpa, sendo esses valores conseqüência do número de sementes formadas nos diferentes tipos de frutos, uma vez que esses micronutrientes desempenham importante papel na maturação das sementes.

Para o manganês, as concentrações nas cascas dos frutos foram estatisticamente superiores (cerca de 3 vezes) às das polpas, onde as maiores concentrações foram observadas nas cascas dos frutos tipo 8, que apresentaram valores estatisticamente superiores aos dos frutos 9 e estes aos do tipo 12.

Na média, os frutos do maracujazeiro doce apresentaram: 452,3 mg de B; 302,5 mg de Cu; $1471 \mathrm{mg}$ de Fe; 167,5 mg de Mn; e 644,1 mg de Zn. Quando comparadas com os valores obtidos por Fernandes et al. (1977) para o maracujazeiro-amarelo, observase que as concentrações dos micronutrientes foram superiores em $233,1 \%$ B, 420,1 \% Cu, 204,8 \% Fe. O Mn e o Zn apresentaram valores $62,5 \%$ e $49 \%$ inferiores aos do maracujá-amarelo, respectivamente.

\section{CONCLUSÕES}

Pelos resultados obtidos, podemos concluir que:

1) Os frutos classificados como tipo 8 são maiores e mais pesados, apresentando maior peso de casca e de polpa.

2) O nitrogênio e o fósforo estão distribuídos em maior quantidade na polpa do que na casca dos frutos, ao inverso do potássio, cálcio, magnésio e enxofre, que estão distribuídos em maior quantidade na casca do que na polpa dos frutos .

3) O boro, cobre, ferro e zinco estão presentes em maior quantidade na polpa do que na casca dos frutos.

4) O manganês está distribuído em maior quantidade na casca do que na polpa dos frutos, com os frutos dos tipos 8 e 9 apresentando valores superiores aos frutos do tipo 12 .

\section{REFERÊNCIAS BIBLIOGRÁFICAS}

ANSELMO, R.M.; JUNQUEIRA, N.T.V.; PINTO, A.C. de Q.; RAMOS, V.H.V.; PEREIRA, A.V.; RANGEL, L.E.P. Caracterização físico-química de dez procedências de maracujá doce. In: CONGRESSO BRASILEIRO DE FRUTICULTURA, 15., 1998, Poços de Caldas. Anais... Poços de Caldas: Sociedade Brasileira de Fruticultura, 1998. p. 577.

BAUMGARTNER, J.G. Nutrição e adubação. In: RUGGIERO, C (Ed.) Maracujá Ribeirão Preto, Legis Summa Ed., p. 86-96. 1987.

FERNANDES, P.D.; OLIVEIRA, G.D. de; RUGGIERO, C.; HAAG, H.P. Extração de nutrientes durante o desenvolvimento do fruto do maracujazeiro-amarelo (Passiflora edulis f. flavicarpa Deg.). O Solo, Piracicaba, v.49, n.1, p. 16-21, 1977.

MALAVOLTA, E., VITTI, G.C., OLIVEIRA, S.A. Avaliação do estado nutricional de plantas. princípios e aplicação, Piracicaba: POTAFOS, 1989. 208p.

OLIVEIRA, J.C.; SALOMÃO, T.A .; RUGGIERO, C. et al. Variações observadas em frutos de Passiflora alata Ait. In: CONGRESSO BRASILEIRO DE OLERICULTURA, 21., CONGRESSO DA SOCIEDADE BRASILEIRA DE FLORICULTURA E PLANTAS ORNAMENTAIS, 2., 1981, Campinas. Proceedings: Campinas, 1982. P. 343-345.

VASCONCELLOS, M.A.S.; CEREDA, C. Cultivo do maracujá doce. In: São José, A. R. (Ed.) Maracujá produção e mercado. Vitória da Conquista: UESB, 1994. P. 71-83.

VASCONCELLOS, M.A.S.; CEREDA, C.; ANDRADE, J. M. de B.; BRANDÃO FILHO, J. U. T. Desenvolvimento de frutos do maracujazeiro doce (Passiflora alata Dryand.), nas condições de Botucatu - SP. Revista Brasileira de Fruticultura, Cruz das Almas, v. 15, n.1, p. 153-158, 1993.

RAIJ, B van; CANTARELLA, H; QUAGGIO, J. A . \& FURLANI, A .M.C. Recomendações de adubação e calagem para o Estado de São Paulo. 2. Ed. Campinas: Instituo Agronômico/Fundação IAC, 1996. 285p. (Boletim técnico, 100) 